\title{
CLOUD-CLOUD COLLISIONS AND FRAGMENTATION
}

\author{
Anthony WHITWORTH and Helen PONGRACIC \\ Department of Physics, \\ University of Wales College of Cardiff, \\ PO Box 913, Cardiff CF1 3TH, \\ Wales, UK.
}

\begin{abstract}
Supersonic head-on collisions between quiescent clouds produce flattened sheets of shocked gas. We derive the condition which the cooling law must satisfy if this sheet is to fragment into protostellar condensations (i.e. gravitationally unstable lumps). If this condition is not satisfied, colliding clouds are likely to be disrupted and dispersed. We show that under the conditions obtaining in GMCs, most cloud-cloud collisions probably do not result in fragmentation.
\end{abstract}

\section{Virial equilibrium}

Consider first a single quiescent cloud of mass $M_{0}$, dimension $L_{0}$, density of hydrogen nuclei in all forms $n_{0}\left(\equiv n_{H I}+2 n_{H_{2}}+\cdots\right)$ and sound speed $a_{0}$. Virial equilibrium requires

$$
a_{0} \sim\left(G M_{0} / L_{0}\right)^{1 / 2} .
$$

If $m$ is the mass associated with one hydrogen nucleus $\left(m \simeq 2.4 \times 10^{-24} \mathrm{gm}\right.$ for population I composition), then

$$
\begin{gathered}
M_{0} \sim L_{0}^{3} n_{0} m ; \\
a_{0} \sim L_{0}\left(G n_{0} m\right)^{1 / 2} .
\end{gathered}
$$

\section{Thermal equilibrium}

We shall assume that the cloud is optically thin to heating and cooling radiation, so that the heating rate per unit volume can be approximated by

$$
\Gamma \sim \Gamma_{r}\left(n / n_{r}\right),
$$


and - at least over a limited range - the cooling rate per unit volume can be approximated by

$$
\Lambda \sim \Gamma_{r}\left(n / n_{r}\right)^{2}\left(a / a_{r}\right)^{\alpha} .
$$

$n_{r}$ and $a_{r}$ are simply reference values for the physical parameters $n$ and $a$. The constant $\Gamma_{r}$ is the same for both $\Gamma$ and $\Lambda$ because we want the reference state $\left(n_{r}, a_{r}\right)$ to be a state of thermal equilibrium. $\Lambda \propto a^{\alpha}$ is roughly equivalent to $\Lambda \propto T^{\alpha / 2}$. Typically $\alpha \sim 3$.

Equating equations (4) and (5) gives the thermal equilibrium condition:

$$
\left(a / a_{r}\right) \sim\left(n / n_{r}\right)^{-1 / \alpha} .
$$

\section{Reference values for physical parameters.}

For the purposes of illustration we adopt $n_{r}=100 \mathrm{~cm}^{-3}$ and $a_{r}=0.5 \mathrm{kms}^{-1}$. Equations (2) and (3) then give $L_{r} \sim 4 \mathrm{pc}$ and $M_{r} \sim 200 M_{\odot}$.

Combining equations (2), (3) and (6), we find that quiescent clouds (i.e. clouds in virial and thermal equilibrium) have,

$$
\begin{gathered}
\left(n_{0} / n_{r}\right) \sim\left(M_{0} / M_{r}\right)^{-2 \alpha /(6+\alpha)} \\
\left(a_{0} / a_{r}\right) \sim\left(M_{0} / M_{r}\right)^{2 /(6+\alpha)} ; \\
\left(L_{0} / L_{r}\right) \sim\left(M_{0} / M_{r}\right)^{(2+\alpha) /(6+\alpha)} .
\end{gathered}
$$

In other words, a more massive cloud has to be hotter and more diffuse if it is to be in virial and thermal equilibrium.

Coincidentally (since we are here assuming only thermal pressure support), equations (7) to (9) with $\alpha \sim 3$ are compatible with Larson's relatic $n s$, viz. $n \propto M^{-6 / 9}$, $a \propto M^{2 / 9}$ and $L \propto M^{5 / 9}$.

\section{General cooling time-scale.}

We shall adopt $\Gamma_{r}=5 \times 10^{-27} \mathrm{erg} \mathrm{cm}^{-3} \mathrm{~s}^{-1}$. This corresponds to a primary ionization rate of $\zeta \sim 10^{-17} \mathrm{~s}^{-1}$. The cooling time-scale is then given by

$$
\begin{gathered}
t^{\text {cool }} \sim \rho a^{2} / \Lambda \sim t_{r}^{\text {cool }}\left(n / n_{r}\right)^{-1}\left(a / a_{r}\right)^{2-\alpha}, \\
t_{r}^{\text {cool }} \sim n_{r} m a_{r}^{2} / \Gamma_{r} \sim 4 \mathrm{Myr} .
\end{gathered}
$$




\section{Collision and expansion time-scales.}

Now consider two identical clouds involved in a head-on collision at relative speed $2 v_{0}=2 \mathcal{M} a_{0}$, where $\mathcal{M}$ is the Mach number. Assuming a strong shock $(\mathcal{M}>>1)$, we know that the density and sound-speed immediately following the shock are $n_{i} \sim 4 n_{0}$ and $a_{i} \sim \mathcal{M} a_{0}=v_{0}$. It follows that the collision time-scale and the timescale on which the flattened sheet expands sideways in the absence of post-shock cooling are roughly equal:

$$
\begin{aligned}
t^{\text {coll }} \sim t^{\exp } \sim & L_{0} / \mathcal{M} a_{0} \sim t_{r}^{\exp } \mathcal{M}^{-1}\left(M_{0} / M_{r}\right)^{\alpha /(6+\alpha)} \\
& t_{r}^{\exp } \sim L_{r} / a_{r} \sim 8 \mathrm{Myr} .
\end{aligned}
$$

\section{Post-shock cooling time-scale.}

Since we expect $\alpha<4$, and since the post-shock cooling regime will be approximately isobaric, cooling will be slowest at high temperatures and the cooling time-scale should be evaluated for the immediate post-shock density and sound-speed, viz.

$$
t_{i}^{\text {cool }} \sim t_{r}^{\text {cool }}\left(n_{i} / n_{r}\right)^{-1}\left(a_{i} / a_{r}\right)^{(2-\alpha)} \sim t_{r}^{\text {cool }} \mathcal{M}^{(2-\alpha)}\left(M_{0} / M_{r}\right)^{4 /(6+\alpha)} .
$$

\section{Fragmentation condition.}

The flattened sheet can only fragment if it does not expand sideways significantly before it cools, i.e. if $t_{i}^{\text {cool }}<<t^{\exp }$ or

$$
\mathcal{M}^{(\alpha-3)}\left(M_{0} / M_{r}\right)^{(\alpha-4) /(6+\alpha)}>>G^{1 / 2}\left(n_{r} m\right)^{3 / 2} a_{r}^{2} \Gamma_{r}^{-1}
$$

Putting $\alpha=3+\epsilon(\epsilon<<1)$, and substituting for the reference parameters, this reduces to

$$
\mathcal{M}^{\epsilon}\left(M_{0} / M_{r}\right)^{-1 / 9}>>0.5 \text {. }
$$

Since the Mach number $\mathcal{M}$ is unlikely to exceed 10 , this inequality can only be satisfied if the clouds are very small and dense $M_{0}<<M_{r}$.

\section{Conclusion.}

Unless the interstellar gas can cool much faster than we have assumed, the majority of cloud-cloud collisions result in disruption and dispersal of the clouds involved. Cloud coalescence is unlikely. Specifically, efficient fragmentation requires that the cooling law of equation (5) has $\alpha \geq 4$ and/or $\Gamma_{r} \geq 10^{-26} \mathrm{ergcm}^{-3} \mathrm{~s}^{-1}$ (corresponding to $\left.\zeta \geq 2 . \times 10^{-17} \mathrm{~s}^{-1}\right)$. 